\title{
Testing of Environmental Satellite Bus-Instrument Interfaces Using Engineering
}

Models

Don Gagnier, Rick Hayner, Michael Roza, Thomas Nosek, Northrop Grumman, Redondo Beach, CA, Andrea Razzaghi, Goddard Space Flight Center, NASA

\section{$\underline{\text { Summary }}$}

This paper discusses the formulation and execution of a laboratory test of the electrical interfaces between multiple atmospheric science instruments and the spacecraft bus that carries them. The testing, performed in 2002, used engineering models of the instruments that will be flown on the Aura spacecraft, and of the Aura spacecraft bus electronics. Aura is one of NASA's Earth Observing System (EOS) Program missions managed by the Goddard Space Flight Center. The test was designed to evaluate the complex interfaces in the spacecraft and instrument command and data handling (C\&DH) subsystems prior to integration of the complete flight instruments on the spacecraft. A problem discovered during (and not before) the flight hardware integration phase can cause significant cost and schedule impacts. The testing successfully surfaced problems and led to their resolution before the full-up integration phase, saving significant cost and schedule time. This approach could be used on future environmental satellite programs involving multiple, complex scientific instruments being integrated onto a bus.

\section{$\underline{\text { Introduction }}$}

Environmental satellites in NASA's EOS earth sciences program such as Aqua (launched successfully in 2002) and Aura (scheduled for launch in 2004) typically carry a number of complex scientific instruments that monitor various facets of the Earth's surface and atmosphere. These instruments ( 8 on Aqua and 4 on Aura) constitute a payload of about $1200 \mathrm{~kg}$. and are integrated onto a spacecraft bus that provides common services including power, thermal control, attitude control, data collection and formatting, and communication. On EOS, the scientific instruments were designed, built and tested by groups other than the spacecraft contractor; the spacecraft contractor manages the overall observatory integration.

The spacecraft C\&DH subsystem provides the interface for commands and telemetry to the instruments, and for storing scientific data from the instruments until the proper times for transmission to the ground. The overall design of the instrument-spacecraft package (the "observatory") involves extensive and often complex interfaces between the instruments and the spacecraft. C\&DH interfaces are the most difficult to analyze and document in advance, and any uncertainties or oversights in this interface lead to problems during observatory integration. Subtle data handling issues involving timing, packet sizing, and the interaction between data streams from multiple instruments come to light during observatory integration can introduce significant delays. These delays can be very costly if when worked during integration while the entire Integration and Test (I\&T) team is standing by. The EOS team developed an approach to testing these interfaces prior to full-scale integration through the use of the engineering models of both 
the spacecraft and instrument $\mathrm{C} \& D H$ subsystems. The objective was schedule and cost risk reduction through identifying and correcting interface problems before committing to full-scale observatory I\&T.

\section{Test Concept}

With the experience on the complexity of instrument-spacecraft data interfaces gained on Aqua, the EOS Aura team developed the concept of a laboratory test of the complete data interface between the spacecraft and the instruments, both individually and jointly as a complete instrument suite. By so doing, instrument-bus compatibility would be demonstrated and interface problems identified and solved prior to actual hardware integration of the instruments on the spacecraft. This concept took advantage of the fact that the spacecraft contractor had developed a laboratory development facility utilizing engineering models of all major spacecraft bus subsystems, including $\mathrm{C} \& \mathrm{DH}$, and that the four instrument developers had independently built either engineering models or interface simulators of their instrument's. The spacecraft team had also completed the flight software used to run the EM processors that control the overall laboratory model spacecraft bus. The team integrated these engineering models for a full-up laboratory representation of the observatory $\mathrm{C} \& D H$ with all four of the instruments. The benefits in time saved during the observatory I\&T phase of the Aura project was significant and outweighed the cost of implementing the test program. The paper describes the formulation of the test concept, identifies the hardware and software involved, discusses how each instrument C\&DH was tested, and how the joint test with all four instruments was conducted.

\section{$\underline{\text { Test Program }}$}

The test program was developed to reduce electrical integration risk of the four scientific instruments on Aura: OMI, TES, MLS, and HIRDLS. These four instruments (basically spectrometers) measure various chemical constituents of Earth's atmosphere, collecting on the order of $50 \mathrm{Gbits}$ of data each 100-minute orbit. Aura carries instrument scientific data for two of the low data rate instruments on the 1553 bus and on a TAXI bus for the two higher rate instruments. Interfaces to be tested included 1553 bus interface characterization, science data generation and inspection, samples of commands and telemetry, operation of stored command sequences, and memory dump and load. Aura carries a solid-state recorder (SSR) where data is stored onboard and dumped once each orbit to NASA's polar ground station. The interface of the instruments through the Formatter Multiplexer Unit (FMU) to the SSR and to the ground communication link is critical to the mission. The test characterized the FMU to TAXI interface and the FMU to 1553 bus interface as well as the operation of the formatter link to the SSR for proper storage and playback. Additionally, a RS-422 link had been provided from the spacecraft inertial reference unit to the TES instrument to allow TES to obtain high-speed real time attitude rate date and this link was tested and characterized during the program. The paper documents the interface problems that were uncovered, and the subsequent fixes and retesting. Cost and schedule risk retirement benefits are identified. 